\title{
A Risk Factor Analysis of Axillary Web Syndrome in Patients After Breast Cancer Surgery: A Single Center Study in Korea
}

\author{
Sangah Jeong, $\mathrm{MD}^{1}$, Byung Joo Song, $\mathrm{MD}^{2}$, Jiyoung $\mathrm{Rhu}, \mathrm{MD}^{3}$, \\ Cheolki Kim, $\mathrm{MD}^{1}$, Sun Im, MD, $\mathrm{PhD}^{1}$, Geun-Young Park, MD, $\mathrm{PhD}^{1}$
}

${ }^{1}$ Department of Rehabilitation Medicine, Bucheon St. Mary's Hospital, College of Medicine, The Catholic University of Korea, Bucheon;

${ }^{2}$ Department of Surgery, Uijeongbu Eulji Medical Center, Eulji University, Uijeongbu;

${ }^{3}$ Department of Surgery, Bucheon St. Mary's Hospital, College of Medicine, The Catholic University of Korea, Bucheon, Korea

Objective To investigate the prevalence and risk factors of axillary web syndrome (AWS) in Korean patients.

Methods This retrospective study included a total of 189 women who underwent breast cancer surgery and received physical therapy between September 2019 and August 2020. We analyzed AWS and the correlation between the patients' demographics, underlying disease, type of surgery and chemotherapy or radiation therapy, and lymphedema.

Results The prevalence of AWS was found to be $30.6 \%$. In the univariable analysis, age, chemotherapy, and hypertension were related to AWS. Finally, the multivariable logistic regression revealed that chemotherapy (odds ratio $[\mathrm{OR}]=2.84$; 95\% confidence interval $[\mathrm{CI}], 1.46-5.53)$ and $\operatorname{HTN}(\mathrm{OR}=2.72 ; 95 \% \mathrm{CI}, 1.18-6.30)$ were the strongest risk factors of AWS.

Conclusion To the best of our knowledge, this was the first study that explored the risk factors of AWS in a Korean population after breast cancer surgery. As almost one-third of patients suffer from AWS after breast cancer surgery, it is essential to closely monitor the development of AWS in patients with hypertension or undergoing chemotherapy.

Keywords Axillary web syndrome, Breast neoplasms, Risk factors, Prevalence, Surgery

Received April 29, 2021; Revised June 28, 2021; Accepted July 12, 2021; Published online October 31, 2021

Corresponding author: Geun-Young Park

Department of Rehabilitation Medicine, Bucheon St. Mary's Hospital, College of Medicine, The Catholic University of Korea, 327 Sosa-ro, Wonmi-gu, Bucheon 14647, Korea. Tel: +82-32-340-2170, Fax: +82-32-340-2173, E-mail: rootpmr@catholic.ac.kr

ORCID: Sangah Jeong (https://orcid.org/0000-0001-7969-6255); Byung Joo Song (https://orcid.org/0000-0002-1461-5263); Jiyoung Rhu (https://orcid. org/0000-0002-9173-5445); Cheolki Kim (https://orcid.org/0000-0002-8385-8197); Sun Im (https://orcid.org/0000-0001-8400-4911); Geun-Young Park (https://orcid.org/0000-0003-0084-2403).

(c) This is an open-access article distributed under the terms of the Creative Commons Attribution Non-Commercial License (http://creativecommons.org/ licenses/by-nc/4.0) which permits unrestricted noncommercial use, distribution, and reproduction in any medium, provided the original work is properly cited. Copyright () 2021 by Korean Academy of Rehabilitation Medicine 


\section{INTRODUCTION}

Axillary web syndrome (AWS) is the formation of fibrotic bands or cords reaching from the axilla to the antecubital fossa and the base of the thumb after breast cancer surgery. The term AWS was initially introduced by Moskovitz et al. [1], and many subsequent studies have investigated its etiology in relation to thrombosis, inflammation, and fibrotic changes in lymphatic vessels and veins. The incidence of AWS reportedly ranges from 6\%-91\% [1-4]. AWS causes shoulder pain during abduction and limits the shoulder's range of motion, thereby interrupting successful rehabilitation after breast cancer surgery. Postoperative radiation therapy is also restricted because the limited shoulder range of motion can impede the patient from assuming the proper posture for radiation therapy. However, there is evidence that adequate physiotherapy can eliminate pain, shorten pain duration, and improve mobility [5].

Several previous studies have investigated the incidence and risk factors of AWS. Some studies revealed that young age and low body mass index (BMI) were risk factors. Underlying diseases like hypertension (HTN) [4] were also found to cause AWS. One study suggested diabetes mellitus (DM) decreases the risk of AWS [4]. Other studies also suggested a link between surgery options or massive lymphatic dissection and AWS [1,4]. Furthermore, some researchers demonstrated that therapeutic options like chemotherapy [6] or radiation therapy [7] were associated with AWS, while a more recent study suggested that breast reconstruction is also a risk factor for AWS [8]. However, many studies reported controversial results and failed to identify consistent risk factors. For instance, one study suggested that low BMI did not correlate with
AWS while arguing that obese patients were more prone to AWS [9]. On the other hand, another study revealed that radiation therapy had a protective effect against AWS [10]. Many recent multivariable analysis studies could not clarify the relationship between age or BMI and AWS. Therefore, to date, the risk factors for AWS have not been accurately established.

To the best of our knowledge, none of the previous studies has explored the risk factors of AWS in Korean patients, although many Korean patients suffer from AWS. Accordingly, in the present study, we aimed to investigate the prevalence and risk factors of AWS in Korean patients, focusing on all assumed risk factors in domestic populations and known risk factors.

\section{MATERIALS AND METHODS}

This was a retrospective chart review study of women who underwent breast cancer surgery in a single university hospital between September 2019 and August 2020. This study was approved by the Institutional Review Board of Bucheon St. Mary's Hospital (No. HC 20RISI0114). The informed consent was waived. Reviewing the patients' medical records, the following data were collected: age, BMI, underlying disease, type of surgery, treatment features, and post-surgical complications (including lymphedema and AWS). The inclusion criteria were as follows: (1) patients who underwent breast cancer surgery for the first time in the period of September 2019-August 2020; and (2) all risk factors were measured. The exclusion criteria were as follows: (1) patients who did not meet the aforementioned inclusion criteria; (2) patients who were lost to follow-up; and (3) patients with missing measurements (Fig. 1).
215 patients with breast cancer surgery from September 2019 to August 2020

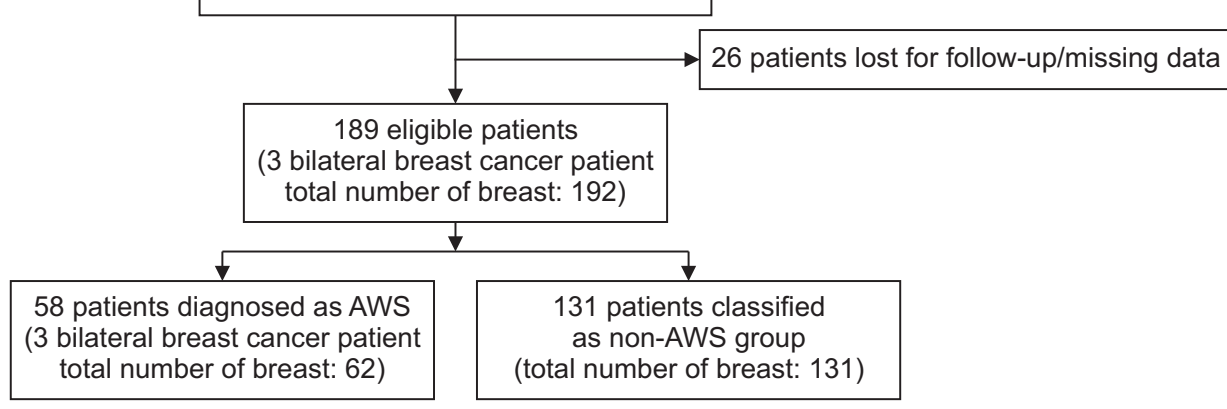

Fig.1. Flow chart of patients' selection. A total of 215 patients were recruited, but 26 patients were lost for follow-up or had missing measurements. AWS, axillary web syndrome. 
Following breast cancer surgery, all patients were required to visit the Department of Rehabilitation 2 weeks after discharge for shoulder exercise education. After patient education, they were asked to visit 4 weeks later for follow-up. The patients with limited shoulder movement, pain, lymphedema, or symptoms of AWS were monitored for a longer period.

The diagnosis of AWS was made during the follow-up period through physical examination. AWS was diagnosed in patients with palpable or visible bands in the ipsilateral side of breast cancer surgery with or without pain in the axilla or upper extremity and limited shoulder movement. The cords usually extend from the axilla along with the medial aspect of the arm to the forearm, reaching even further to the base of the thumb. Trained rehabilitation doctors evaluated these patients with ultrasonography for further evaluation of AWS. The assessment was performed using a high-frequency (3-12 MHz) linear array transducer L3-12A (Samsung Medison Co. Ltd, Seoul, Korea), tracing along the suspected cord with moderate pressure to detect AWS deep within the subcutaneous layer. The sonographic findings of anechoic or hypoechoic cords with surrounding hyperechoic edges or structures were regarded as AWS [11] (Fig. 2).

Based on previous studies, the following risk factors were collected and analyzed: demographic data (age, BMI), underlying disease (DM, HTN), surgery options (type of operation, extent of lymph node dissection, reconstruction), other treatment options (radiation therapy, chemotherapy), and lymphedema.

The patients were divided into two groups: above 50 years and under 50 years. Based on World Health Organization (WHO) guidelines for BMI in Asian populations
[12], the patients with BMI lower than $18.5 \mathrm{~kg} / \mathrm{m}^{2}$ were classified as underweight, $18.5-23 \mathrm{~kg} / \mathrm{m}^{2}$ as normal, and above $23 \mathrm{~kg} / \mathrm{m}^{2}$ as overweight. Surgery types were divided into breast-conserving surgery (BCS), simple or total mastectomy, and modified radical mastectomy. BCS is a surgical procedure that removes the primary breast tumor with clean margins in the surrounding normal breast parenchyma in a cosmetically acceptable manner. Mastectomy refers to the complete removal of the mammary gland, including the nipple and areola [13]. Currently, mastectomy has evolved to skin-sparing or nipple-sparing mastectomy [14]. The modified radical mastectomy removes the mammary gland, nipple, and areola, with the addition of a complete axillary lymph node dissection [13]. The pectoralis minor muscle may also be removed depending on the extent of tumor invasion or surgery technique [15]. Lymph node dissection extent was classified into simple lymph node dissection and axillary lymph node dissection. Reconstruction operation included tissue expander followed by implant placement, immediate implant placement, autologous tissue transplantation, and a procedure that incorporated both breast implants and autologous tissue transplantation. All chemotherapy regimens, neoadjuvant, adjuvant chemotherapy, and radiation therapy before and after the operation were included if these had been administered before the development of AWS. Lymphedema was evaluated through arm circumference measurement and ultrasonography. Lymphoscintigraphy was also performed on patients with suspected lymphedema. If a patient underwent a bilateral operation, we selected the contralateral side where the AWS occurred; otherwise, the contralateral side that underwent a wider extent of
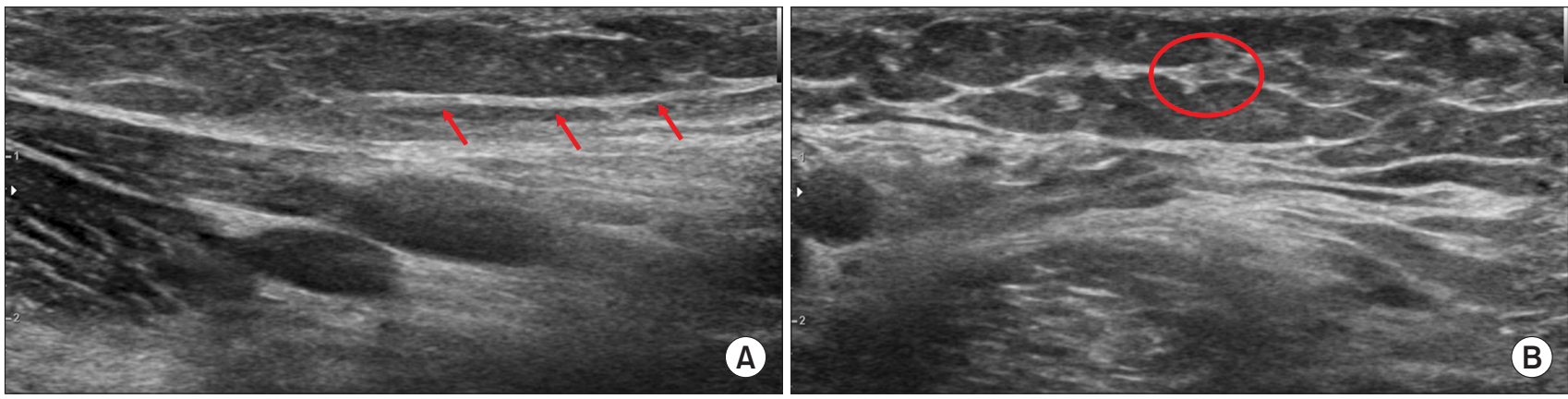

Fig. 2. Ultrasonography image of axillary web syndrome showing anechoic or hypoechoic cord with surrounding hyperechoic edges or structures in (A) longitudinal view (arrow) and (B) transverse view. 
surgery was chosen.

Categorical variables were analyzed using the chisquare test or Fisher exact test. Univariable logistic regression and stepwise multivariable logistic regression analyses were performed. The analysis was run using SAS system version 9.4 (SAS Institute Inc., Cary, NC, USA). A significance level of 0.05 was adopted. The odds ratio was calculated to evaluate the degree of relevance with a con- fidence interval (CI) of $95 \%$.

\section{RESULTS}

Between September 2019 and August 2020, a total of 189 eligible patients were recruited. The participants' baseline characteristics are summarized in Table 1. AWS was found in 58 (30.6\%) patients.

Table 1. Demographics and clinical variables of study participants

\begin{tabular}{|c|c|c|c|c|}
\hline & AWS Yes $(n=58)$ & AWS No $(n=131)$ & Total $(n=189)$ & p-value \\
\hline Age (yr) & $51.07 \pm 9.88$ & $55.84 \pm 10.54$ & $54.37 \pm 10.54$ & $0.003^{*}$ \\
\hline \multicolumn{5}{|l|}{ BMI $\left(\mathrm{kg} / \mathrm{m}^{2}\right)$} \\
\hline$>23$ & $22(37.93)$ & $91(69.47)$ & $113(59.78)$ & \\
\hline $18.5-23$ & $33(56.90)$ & $40(30.53)$ & $73(41.26)$ & \\
\hline$<18.5$ & $3(5.17)$ & $0(0)$ & $3(1.58)$ & $<0.001^{*}$ \\
\hline \multicolumn{5}{|l|}{ Operation } \\
\hline BCS & $29(50.00)$ & $63(48.09)$ & $92(48.67)$ & \\
\hline Mastectomy & $21(36.21)$ & $40(30.53)$ & $61(32.27)$ & \\
\hline MRM & $8(13.79)$ & $28(21.37)$ & $36(19.04)$ & 0.439 \\
\hline \multicolumn{5}{|c|}{ Lymph node dissection } \\
\hline No & $0(0)$ & $1(0.76)$ & $1(0.50)$ & \\
\hline SLND & 38 (65.52) & $82(62.60)$ & $120(63.49)$ & \\
\hline ALND & $20(34.48)$ & $48(36.64)$ & $68(35.97)$ & 0.909 \\
\hline \multicolumn{5}{|l|}{ Reconstruction } \\
\hline No & 37 (63.79) & $91(69.47)$ & $128(67.72)$ & \\
\hline Yes & $21(36.21)$ & $40(30.53)$ & $61(32.27)$ & 0.441 \\
\hline \multicolumn{5}{|l|}{ Chemotherapy } \\
\hline No & $33(56.90)$ & 39 (29.77) & 72 (38.09) & \\
\hline Yes & $25(43.10)$ & $92(70.23)$ & $117(61.90)$ & $<0.001^{*}$ \\
\hline \multicolumn{5}{|c|}{ Radiation therapy } \\
\hline No & 57 (98.28) & 74 (56.49) & $131(69.31)$ & \\
\hline Yes & $1(1.72)$ & $57(43.51)$ & $58(30.68)$ & $<0.001^{*}$ \\
\hline \multicolumn{5}{|l|}{$\mathrm{DM}$} \\
\hline No & 54 (93.10) & $107(81.68)$ & $161(85.18)$ & \\
\hline Yes & $4(6.90)$ & $24(18.32)$ & $28(14.81)$ & $0.041^{*}$ \\
\hline \multicolumn{5}{|l|}{ Hypertension } \\
\hline No & 49 (84.48) & 80 (61.07) & $129(68.25)$ & \\
\hline Yes & $9(15.52)$ & $51(38.93)$ & $60(31.74)$ & $0.001^{*}$ \\
\hline \multicolumn{5}{|l|}{ Lymphedema } \\
\hline No & 53 (91.38) & $109(83.21)$ & $162(85.71)$ & \\
\hline Yes & $5(8.62)$ & $22(16.79)$ & $27(14.28)$ & 0.138 \\
\hline
\end{tabular}

Values are presented as mean \pm standard deviation or number (\%).

AWS, axillary web syndrome; BMI, body mass index; BCS, breast-conserving surgery; MRM, modified radical mastectomy; SLND, sentinel lymph node dissection; ALND, axillary lymph node dissection; DM, diabetes mellitus.

${ }^{*} \mathrm{p}<0.05$ by chi-square test or Fisher exact test. 
The average time to AWS onset was 33.61 days, consistent with other studies, which revealed that AWS occurred in approximately 12-30 days [3]. One study reported that $86.1 \%$ of the cords appeared 30 days after surgery, with most occurring by the seventh day (66.1\%) [4]. The mean age was $54.37 \pm 10.54$ years. The average BMI of the patients was $24.34 \pm 3.65 \mathrm{~kg} / \mathrm{m}^{2}$, which is slightly higher than the normal BMI boundary. The average BMI of patients with AWS was $22.83 \mathrm{~kg} / \mathrm{m}^{2}$, which was lower than the average of $25.02 \mathrm{~kg} / \mathrm{m}^{2}$ for patients without AWS.

In the univariable logistic regression, age, chemotherapy, and HTN showed significant differences between the groups (Table 2). The patients younger than 50 years were 0.37 times ( $95 \% \mathrm{CI}, 0.19-0.71 ; \mathrm{p}=0.003$ ) less likely to develop AWS than those older than 50 years. The patients who underwent chemotherapy were 3.11 times (95\% CI, $1.64-5.90 ; \mathrm{p}<0.001)$ more likely to have AWS than the non-AWS group. The patients with HTN were 3.47 times (95\% CI, 1.57-7.66; $\mathrm{p}=0.002$ ) more likely to develop AWS.
The multivariable logistic regression was used to analyze the variables that showed significant differences in the univariable logistic regression (Table 2). Chemotherapy and HTN were the most significant risk factor for AWS. The patients who underwent chemotherapy were 2.84 times (95\% CI, 1.46-5.53; $\mathrm{p}=0.002$ ) more likely to develop AWS. Finally, patients with HTN were 2.72 times (95\% CI, 1.18-6.30; $\mathrm{p}=0.01$ ) more likely to have AWS than the patients without HTN.

\section{DISCUSSION}

This study aimed to investigate the prevalence and risk factors of AWS in Korean patients. According to our results, AWS was observed in $30.6 \%$ of patients. A systematic review [5] discussed that the incidence or prevalence of AWS varied depending on the race or study design. Retrospective studies including all types of surgery and lymph node dissection showed that the prevalence of

Table 2. Univariable and multivariable logistic regression for risk factors associated with axillary web syndrome

\begin{tabular}{|c|c|c|c|c|c|c|}
\hline & \multicolumn{3}{|c|}{ Univariable } & \multicolumn{3}{|c|}{ Multivariable } \\
\hline & $\mathbf{O R}$ & 95\% CI & p-value & OR & $95 \%$ CI & p-value \\
\hline Age (ref. age $>50 \mathrm{yr}$ ) & 0.37 & $0.1-0.71$ & $0.003^{*}$ & 0.522 & $0.25-1.05$ & 0.068 \\
\hline \multicolumn{7}{|l|}{$<50$} \\
\hline \multicolumn{7}{|c|}{ BMI $\left(\right.$ ref. BMI $\left.>23 \mathrm{~kg} / \mathrm{m}^{2}\right)$} \\
\hline $18.5-23$ & 0.29 & $0.15-0.56$ & 0.970 & & & \\
\hline$<18.5$ & $<0.001$ & $<0.001,>999.99$ & 0.965 & & & \\
\hline \multicolumn{7}{|l|}{ Operation (ref. BCS) } \\
\hline Mastectomy & 0.87 & $0.44-1.74$ & 0.296 & & & \\
\hline MRM & 1.61 & $0.65-3.96$ & 0.214 & & & \\
\hline \multicolumn{7}{|c|}{ Lymph node dissection (ref. SLND) } \\
\hline ALND & 1.12 & $0.58-2.12$ & 0.972 & & & \\
\hline \multicolumn{7}{|c|}{ Reconstruction (ref. not performed) } \\
\hline Yes & 0.77 & $0.40-1.48$ & 0.442 & & & \\
\hline \multicolumn{7}{|c|}{ Chemotherapy (ref. not performed) } \\
\hline Yes & 3.11 & $1.64-5.90$ & $<0.001^{*}$ & 2.84 & $1.46-5.53$ & $0.002^{*}$ \\
\hline \multicolumn{7}{|l|}{ DM (ref. absence) } \\
\hline Yes & 3.02 & $1.00-9.16$ & 0.05 & & & \\
\hline \multicolumn{7}{|c|}{ Hypertension (ref. absence) } \\
\hline Yes & 3.47 & $1.57-7.66$ & $0.002^{*}$ & 2.72 & $1.18-6.30$ & $0.018^{*}$ \\
\hline \multicolumn{7}{|c|}{ Lymphedema (ref. absence) } \\
\hline Yes & 2.13 & $0.76-5.96$ & 0.145 & & & \\
\hline
\end{tabular}

OR, odds ratio; CI, confidence interval; BMI, body mass index; BCS, breast-conserving surgery; MRM, modified radical mastectomy; SLND, sentinel lymph node dissection; ALND, axillary lymph node dissection; DM, diabetes mellitus. ${ }^{*} \mathrm{p}<0.05$. 
AWS was $19 \%$ [16] and $29.4 \%$ [10], respectively, similar to our study. A study of 110 Korean revealed that AWS developed in $9 \%$ of patients [17], but this study only included clinical T1-2 stage patients, which may have led to the relatively lower prevalence compared to our study. The difference in incidence or prevalence might be due to the difference in study design and AWS detection methods.

The results of our study suggest that the risk of AWS increases with age and in those with HTN and who underwent chemotherapy. The multivariable logistic regression analysis showed that chemotherapy and HTN were the most important factors for the development of AWS.

Many previous studies suggested that younger age might be a risk factor for AWS $[3,18]$. However, these studies analyzed the data using univariable analysis only. Recent multivariable logistic regression studies have revealed that age was not a risk factor for AWS $[9,19]$. In contrast, the average age above 50 years was found to be a strong predictor of AWS in our study. However, considering other confounding factors, age was not statistically significant in the final statistical model.

Furthermore, many previous studies reported that a lower BMI increased the risk of AWS $[2,3,18]$. However, most of these studies relied on the results of univariable analyses that did not consider other confounding variables. Many recent multivariable logistic studies revealed that BMI has no relationship with AWS $[4,9,20]$. In our study, the average BMI of the patients with AWS was 22.83 $\mathrm{kg} / \mathrm{m}^{2}$, while that in the contrast group was $25.02 \mathrm{~kg} / \mathrm{m}^{2}$,

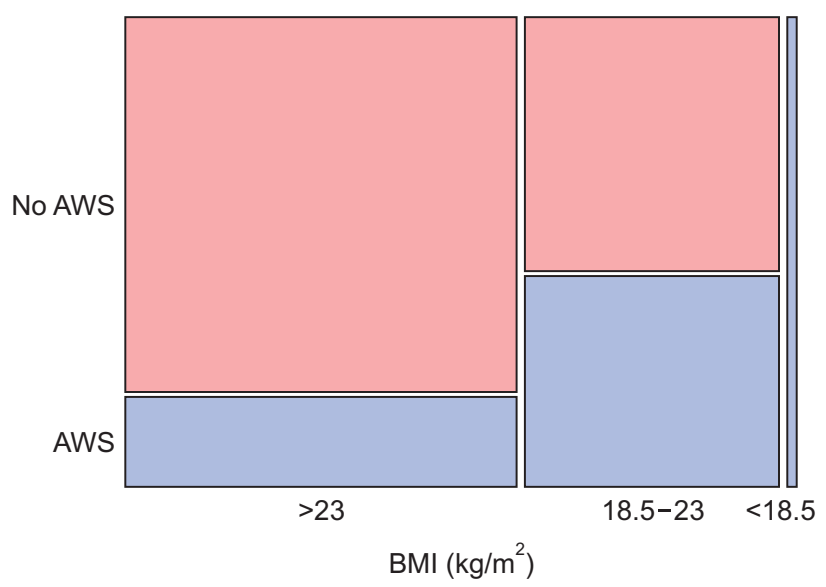

Fig. 3. Cochran-Armitage trend test of body mass index (BMI) and axillary web syndrome (AWS). suggesting a lower BMI tendency in the AWS group. We divided the patients depending on their BMI following the WHO Asian classification and used logistic regression for data analysis. In our results, BMI was not a statistically significant risk factor. This can be explained by the fact that the increased amount of adipose tissue in the axilla region in obese patients might hinder the detection of cords, possibly explaining the higher risk of AWS among patients with lower BMI in previous studies.

While we failed to find a correlation between BMI and AWS in the final model, our results confirmed that AWS patients showed a tendency for low BMI in the CochranArmitage trend test (Fig. 3). As our study included only three underweight BMI patients, and the average BMI of our patients was $24.34 \mathrm{~kg} / \mathrm{m}^{2}$, this might suggest that BMI has no statistically significant difference. Further research including an even BMI distribution might be needed.

For the underlying disease, we failed to establish a relation between DM and AWS. However, HTN was associated with AWS. The patients with HTN were 2.72 times more likely to develop AWS than those with no HTN. A prospective study evaluating the correlation between vascular change and AWS [21] discovered diameter restriction in the brachial artery and increased mean flow velocity of axillary artery in AWS patients, suggesting that AWS accompanies vascular and lymphatic changes. In hypertensive patients, structural, mechanical, or functional changes may reduce the lumen diameter of small arteries and arterioles, increasing blood flow velocity [22]. In this context, HTN may aggravate artery flow limitation and diameter reduction changes in AWS. Further research is needed to confirm this hypothesis.

Several previous studies revealed that a more extensive range of operation types and lymph node dissection damages lymphatic structures, leading to the development of AWS $[4,19]$. However, our results showed that operation type and lymph node dissection extent were not relevant to AWS. Some cases reported AWS in transaxillary robotic thyroidectomy without lymph node dissection or following axillary furunculosis $[23,24]$. From this evidence, it can be inferred that AWS occurs in any procedure that disrupts the lymphatic or vascular structures of the axillary area and is not based on the direct lymph node dissection extent.

A recent study revealed that breast reconstruction increases the risk of AWS [8]. However, in our study, breast 
reconstruction and AWS were not related. Chemotherapy is one of the widely used options for breast cancer treatment and can be done before or after surgery. In this study, chemotherapy was found to be one of the strongest risk factors for AWS. Patients who received chemotherapy had a 2.84-times higher incidence of AWS than the patients who did not. Histopathological changes in blood and lymphatic vessels in the skin and subcutaneous tissue of breast cancer patients taking chemotherapy showed more angiogenesis and microvessel density [25]. These changes could reinforce the inflammation and fibrotic changes accompanying AWS.

Patients who underwent radiation therapy showed statistical significance with AWS in Fisher exact test. Although statistical meaningful results were obtained, this finding could not be considered clinically meaningful because only one person received radiation therapy before AWS. Radiation therapy was excluded from the logistic regression analysis.

AWS and lymphedema share a similar pathophysiology since both are caused by lymphatic damage and inflammatory reactions, which are believed to be related to each other [26]. One study revealed that lymphedema is a risk factor of AWS [6]. A retrospective study by Ryan et al. [20] showed that patients with AWS had a 44\% greater risk of developing lymphedema. On the contrary, Wariss et al. [27] stated that AWS is not a risk factor for lymphedema after 10 years of follow-up. The relationship between AWS and lymphedema has yet to be confirmed in a systematic review [5]. Our study showed no significant relationship between lymphedema and AWS. This may be because lymphedema is caused by fluid congestion and chronic inflammation due to impaired lymphatic drainage [28], whereas AWS is thought to be caused by regional lymphatic injury. Also, AWS resolution occurs mostly within 3 weeks to 3 months following surgery, although some studies showed that natural resolution occurs after more than 3 months [5]. Thus, this natural improvement is unlikely to cause lymphedema with chronic flow restriction. Further studies are needed for this hypothesis.

Our study had several limitations. First, since this was a retrospective study, selection bias might have occurred. Second, this was a single-center study that included only some available regional patient groups. Third, in some variables, the number of patients recruited was small. In the case of BMI, only three underweight persons were included. This uneven distribution of BMI in our population might have affected the study results. Also, while radiation therapy showed a statistically significant difference in univariable analysis, this might be the result of a small number of patients. In the 58 patients with AWS, only one underwent radiation therapy. To correct this error, we excluded radiation therapy from the final statistical analysis. Fourth, although we suggested risk factors for AWS, we could not confirm why these risk factors increased the risk of AWS. Future multi-center studies with more extensive risk factor analysis are necessary.

In summary, chemotherapy and HTN were the strongest risk factors for AWS development. This suggests that systemic changes accompanying vascular or lymphatic changes, in addition to some regional insult in the axilla itself, might increase the risk of AWS. As AWS can limit successful post-operative rehabilitation, patients should be followed up closely to check for AWS development.

\section{CONFLICT OF INTEREST}

No potential conflict of interest relevant to this article was reported.

\section{AUTHOR CONTRIBUTION}

Conceptualization: Park GY. Methodology: Im S, Kim CK, Rhu JY, Song BJ. Formal analysis: Jeong SA. Project administration: Park GY. Visualization:Jeong SA, Kim CK. Writing - original draft: Jeong SA. Writing - review and editing: Park GY, Im S. Approval of final manuscript: all authors.

\section{REFERENCES}

1. Moskovitz AH, Anderson BO, Yeung RS, Byrd DR, Lawton TJ, Moe RE. Axillary web syndrome after axillary dissection. Am J Surg 2001;181:434-9.

2. Leidenius M, Leppanen E, Krogerus L, von Smitten K. Motion restriction and axillary web syndrome after sentinel node biopsy and axillary clearance in breast cancer. Am J Surg 2003;185:127-30.

3. Torres Lacomba M, Mayoral Del Moral O, Coperias Zazo JL, Yuste Sanchez MJ, Ferrandez JC, Zapico Goni A. Axillary web syndrome after axillary dissection in breast cancer: a prospective study. Breast Cancer Res 
Treat 2009;117:625-30.

4. Figueira P, Haddad C, de Almeida Rizzi S, Facina G, Nazario A. Diagnosis of axillary web syndrome in patients after breast cancer surgery: epidemiology, risk factors, and clinical aspects: a prospective study. Am J Clin Oncol 2018;41:992-6.

5. Yeung WM, McPhail SM, Kuys SS. A systematic review of axillary web syndrome (AWS). J Cancer Surviv 2015;9:576-98.

6. de Sire A, Losco L, Cisari C, Gennari A, Boldorini R, Fusco N, et al. Axillary web syndrome in women after breast cancer surgery referred to an Oncological Rehabilitation Unit: which are the main risk factors? A retrospective case-control study. Eur Rev Med Pharmacol Sci 2020;24:8028-35.

7. Brunelle CL, Roberts SA, Shui AM, Gillespie TC, Daniell KM, Naoum GE, et al. Patients who report cording after breast cancer surgery are at higher risk of lymphedema: results from a large prospective screening cohort. J Surg Oncol 2020;122:155-63.

8. Huang HC, Liu HH, Yin LY, Weng CH, Fang CL, Yang CS. High incidence of axillary web syndrome among breast cancer survivors after breast reconstruction. Breast Care (Basel) 2020;15:366-71.

9. Fukushima KF, Carmo LA, Borinelli AC, Ferreira CW. Frequency and associated factors of axillary web syndrome in women who had undergone breast cancer surgery: a transversal and retrospective study. Springerplus 2015;4:112.

10. Severeid K, Simpson J, Templeton B, York R, HummelBerry K, Leiserowitz A. Lymphatic cording among patients with breast cancer or melanoma referred to physical therapy. Rehabil Oncol 2007;25:8-13.

11. Mullen LA, Harvey SC. Review of axillary web syndrome: What the radiologist should know. Eur J Radiol 2019;113:66-73.

12. WHO Expert Consultation. Appropriate body-mass index for Asian populations and its implications for policy and intervention strategies. Lancet 2004;363:15763.

13. Townsend CM, Beauchamp DR, Evers MB, Mattox KL. Sabiston textbook of surgery: the biological basis of modern surgical practice. 20th ed. Philadelphia, PA: Elsevier; 2016.

14. Jones C, Lancaster R. Evolution of operative technique for mastectomy. Surg Clin North Am 2018;98:835-44.
15. Bland KI, Suzanne Klimberg V. Master techniques in general surgery: breast surgery. Philadelphia, PA: Lippincott Williams \& Wilkins; 2011.

16. Wyrick SL, Waltke LJ, Ng AV. Physical therapy may promote resolution of lymphatic cording in breast cancer survivors. Rehabil Oncol 2006;24:29-34.

17. Huh SJ, Yang JH, Park W, Nam SJ, Kim JH. Axillary web syndrome after sentinel node biopsy and axillary lymph node dissection during the conservative treatment of early breast cancer. J Breast Cancer 2005;8:9498.

18. Ramirez-Parada K, Garay-Acevedo D, Mella-Abarca W, Petric-Guajardo M, Sanchez-Rojel C, McNeely ML, et al. Axillary web syndrome among Chilean women with breast cancer: incidence and possible predisposing factors. Support Care Cancer 2020;28:2941-7.

19. Bergmann A, Mendes VV, de Almeida Dias R, do Amaral E Silva B, da Costa Leite Ferreira MG, Fabro EA. Incidence and risk factors for axillary web syndrome after breast cancer surgery. Breast Cancer Res Treat 2012;131:987-92.

20. Ryans K, Davies CC, Gaw G, Lambe C, Henninge M, VanHoose L. Incidence and predictors of axillary web syndrome and its association with lymphedema in women following breast cancer treatment: a retrospective study. Support Care Cancer 2020;28:5881-8.

21. Furlan C, Matheus CN, Jales RM, Derchain S, Sarian LO. Vascular alterations in axillary and brachial vessels in patients with axillary web syndrome after breast cancer surgery. Lymphat Res Biol 2018;16:28793.

22. Schiffrin EL. Vascular remodeling in hypertension: mechanisms and treatment. Hypertension 2012;59:367-74.

23. Kim JH, Park JW, Gong HS. Axillary web syndrome after transaxillary robotic thyroidectomy. J Robot Surg 2014;8:281-3.

24. Rashtak S, Gamble GL, Gibson LE, Pittelkow MR. From furuncle to axillary web syndrome: shedding light on histopathology and pathogenesis. Dermatology 2012;224:110-4.

25. Nakagawa A, Fujimoto H, Nagashima T, Sangai T, Takada M, Masuda T, et al. Histological features of skin and subcutaneous tissue in patients with breast cancer who have received neoadjuvant chemotherapy and their relationship to post-treatment edema. 
Breast Cancer 2020;27:77-84.

26. Lee KW, Kim SB, Lee JH, Kim YS. Effects of extracorporeal shockwave therapy on improvements in lymphedema, quality of life, and fibrous tissue in breast cancer-related lymphedema. Ann Rehabil Med 2020;44:386-92.

27. Wariss BR, Costa RM, Pereira AC, Koifman RJ, Berg- mann A. Axillary web syndrome is not a risk factor for lymphoedema after 10 years of follow-up. Support Care Cancer 2017;25:465-70.

28. Grada AA, Phillips TJ. Lymphedema: pathophysiology and clinical manifestations. J Am Acad Dermatol 2017;77:1009-20. 